2. Magnetic Effects 


\title{
LOW ENERGY LINES IN SPECTRA OF GAMMA BURSTS
}

\author{
G.S. Bisnovatyi-Kogan, A.F. Illarionov \\ Space Research Institute, Moscow, USSR
}

\begin{abstract}
We connect the phenomenon of gamma ray bursts with nuclear explosions on the old neutron stars. The matter of the neutron star in the non-equilibrium layer at depths of $30 \mathrm{~m} \leq h \leq 100 \mathrm{~m}$ consists of superheavy $(A \geq 300)$ nuclei with a surplus of neutrons $(A / Z=3 \div 4)$. These nuclei are metastable and exist only at high pressure. After the starquake some of the matter from non-equilibrium layer may move upwards and its nuclei become unstable. The $\beta$-decay is followed by a chain reaction of fission and nuclear explosion. The gamma ray burst is observed as radiation of the star surface heated to high temperature. Some mass may be ejected, forming expanding cloud. It consists mainly of the iron $\mathrm{Fe}^{56}$ with small $(\leq 1 \%)$ additions of heavy elements $(\mathrm{Ba}, 1, \ldots)$ arising from the fission. The passage of stellar gamma radiation through the expanding plasma clouds leads to the formation of short-lived spectral features. Strong absorption of the soft gamma rays on $\mathrm{K}$-electrons of $\mathrm{Fe}^{56}$ must be observed in the early stages. The gamma quanta with energies $\varepsilon=40-70 \mathrm{keV}$ beyond the $\mathrm{K}$-edge of the heavy elements $(\mathrm{Ba}, \mathrm{I}, \ldots)$ are absorbed in the later stages. A wide $\kappa_{\alpha}$ line $\left(\varepsilon_{\alpha}=30 \mathrm{keV}\right)$ appears simultaneously. The free-free emission of expanding hot plasma cloud may be observed as a short flash in optical band.
\end{abstract}

\section{INTRODUCTION}

The short rising time of the cosmic gamma ray bursts $\left(\tau \leq 2 \times 10^{-4} \mathrm{~s}\right)$ and hard spectrum $\left(\varepsilon_{0}\right.$ $=0.1 \div 1 \mathrm{MeV}$ ) could not be explained by the model of thermonuclear explosion in the matter of neutron star envelope gained by accretion in a binary system. Really this model gives larger rising times $(\tau \geq 0.1 \mathrm{~s})$ softer $\left(\varepsilon_{0}=5 \div 10 \mathrm{keV}\right)$ spectra with a maximum in $\mathrm{X}$ ray region. The optical companions needed for accretion are not found in gamma ray burst sources.

We follow an alternative model based on nuclear explosions (fission), which happen in the undersurface layers of old $\left(\geq 10^{7} \mathrm{yr}\right)$ single neutron stars. A very short rising time is possible to explain with this model. The analysis of spectral features of gamma ray bursts permits us to follow the process of the explosion and of the interaction of gamma radiation with the matter ejected from the neutron star by the shock wave.

Observations of gamma ray bursts in the "Konus" experiment have shown in some 
events the presence of spectral features in the energy range (see Fig. 1) $\varepsilon=30-70 \mathrm{keV}$ (Mazets et al., 1980, 1981; Golenetskii et al., 1983).

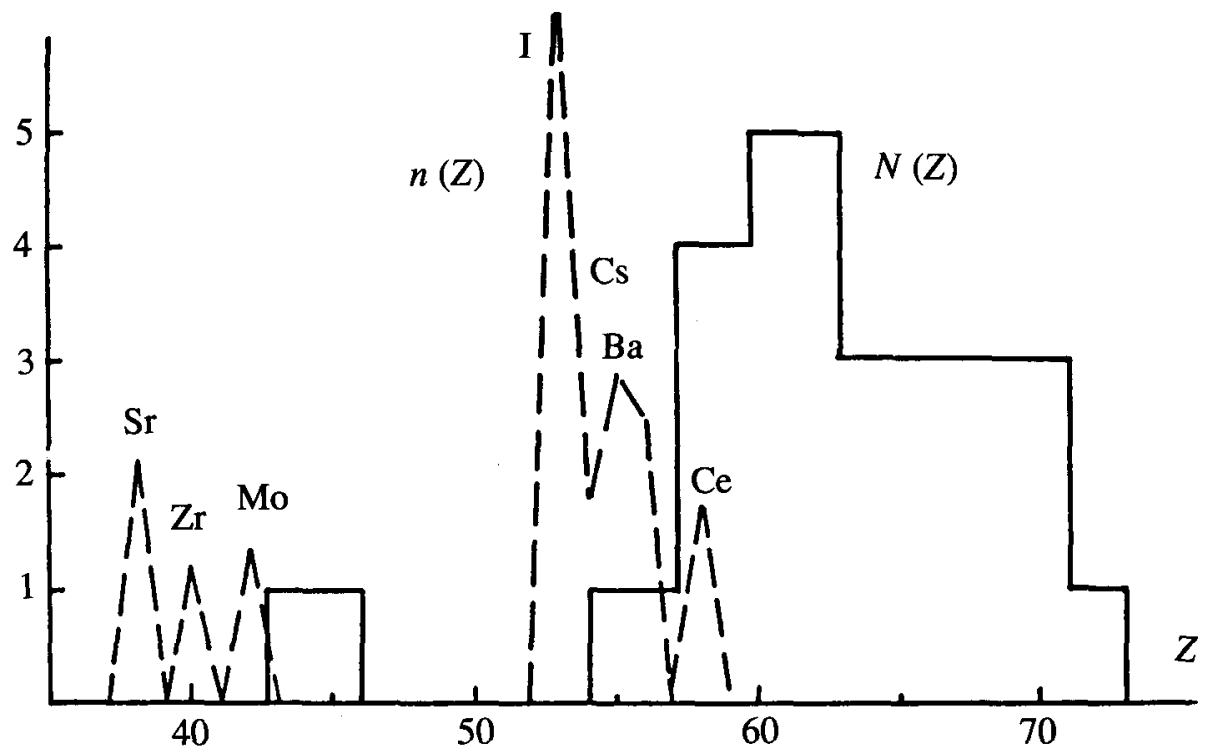

Fig. 1. Histogram of the distribution of the number of gamma ray bursts $N$ on the energy $\varepsilon$, reduced to the axis $Z$ according to relation $\varepsilon=13.6 Z 2 \mathrm{eV}$ (full line) .

The histogram $n(Z)$ of the element production in the fission of $\mathrm{U}^{235}$ as a function of the atomic number $Z$ (dashed line).

The attempt to interpret these lines by cyclotron absorption (Mazets and Golenetskii, 1987) leads to unlikely large values of magnetic field

$$
H \approx \varepsilon \frac{m c}{e h} \approx(3-7) 10^{12} \mathrm{Gs}
$$

The majority of the lines (70\%), see Fig 1 , have energies $\varepsilon>50 \mathrm{keV}$, corresponding to $H>5 \times 10^{15} \mathrm{Gs}$. (An account of red shift on the surface of neutron star increases the estimated values of $H$.) Such values are too large in comparison with the fields (Rahakrishnan, 1982) of young neutron stars-radiopulsars $\left(H \approx 10^{12} \mathrm{Gs}\right)$. Isotropic distribution of the gamma ray burst sources permits us to connect them with close $(\leq 100 \mathrm{pc})$, old neutron stars with moderate magnetic fields $\left(H \approx 10^{8} \div 10^{10} \mathrm{Gs}\right)$. The absence of the motion of absorbance features across the spectrum and their rapid disappearance against the continuum background of gamma ray burst spectrum cannot be explained satisfactorily by the cyclotronic hypothesis of origin of these lines. 


\section{PHOTOABSORPTION OF GAMMA RAYS BY HEAVY ELEMENTS WITH}

$Z=40 \div 60$

Rapid cooling of matter in conditions of high density $p \approx 10^{11} \mathrm{~g} / \mathrm{cm}^{3}$ leads to the formation of superheavy nuclei near the neutron drip line with atomic weight $A \approx 4 Z$ and the charge

$$
Z=7\left[33-0.511\left(\rho / 10^{6} \mu_{\mathrm{e}}\right)^{13}\right], \mu_{\mathrm{e}} \approx 4
$$

(Bisnovatyi-Kogan and Chechetkin, 1974). These nuclei are stable in the non-equilibrium layer at densities

$$
6 \times 10^{10} \mathrm{~g} / \mathrm{cm}^{3} \approx \rho 1<\rho<\rho_{2} \approx 10^{12} \mathrm{~g} / \mathrm{cm}^{3}
$$

At low densities $\rho<\rho 1$ the superheavy nuclei emit electrons and antineutrinos ( $\beta$-decay), become unstable, divide into two non-equal parts (like the fission of $\mathrm{U}^{235}$ ) and a chain reaction occurs (Bisnovatyi-Kopgan and Chechetkin, 1983). We believe that starquakes induce the movement of superheavy nuclei to lower density regions and thus fission chain reactions (see Fig. 2).

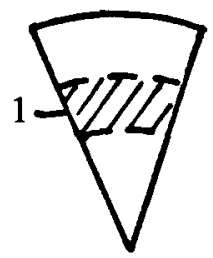

a

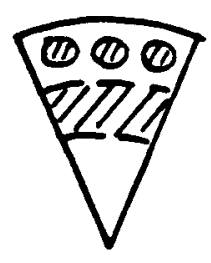

b

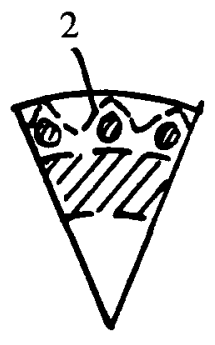

c
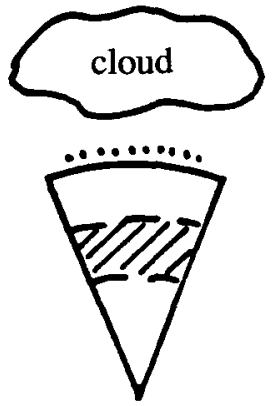

d

Fig. 2. Schematic picture of gamma ray burst formation

(a) non-equilibrium layer (1) in the static envelope

(b) after starquake

(c) after nuclear explosion (2) - the shock waves

(d) hot surface and expanding cloud 
Table 1. The charges and ionization potentials of the most abundant nuclei formed in the fission

\begin{tabular}{|c|c|c|c|c|c|c|c|c|}
\hline & $\mathrm{Fe}$ & $\mathrm{Sr}$ & $\mathrm{Zr}$ & $\mathrm{Mo}$ & $\mathrm{I}$ & $\mathrm{Cs}$ & $\mathrm{Ba}$ & $\mathrm{Ce}$ \\
\hline$Z$ & 26 & 38 & 40 & 42 & 53 & 55 & 56 & 58 \\
\hline$I \mathrm{keV}$ & 9.3 & 20 & 22 & 24 & 38 & 41 & 43 & 46 \\
\hline
\end{tabular}

The shockwave from the nuclear explosion comes to the surface and ejects some matter in a form of an expanding cloud. Its radius $R \approx v t$ increases with a velocity $v \approx$ $\sqrt{ }\left(2 G M / R_{0}\right) \approx(0.1-0.5) c$. The composition of the cloud is mainly iron $\mathrm{Fe}^{56}$ with small ( $\leq$ $1 \%$ ) additions of the products of nuclear fission (see Table 1). The initial spectrum of gamma radiation is distorted due to the passage of gamma rays through the cloud to the observer. Initially the optical depth of the iron cloud is high beyond the K-edge $\varepsilon \geq I_{\mathrm{Fe}}=$ $13.6 Z^{2} \mathrm{eV} \approx 9.3 \mathrm{keV}$ because of photoabsorption. The cloud is transparent only for the hard component of gamma radiation (see Fig. 3), because the cross-section of absorption is high only near the edge and decreases with energy as

$$
a(\varepsilon) \approx \frac{a_{\mathrm{H}}}{Z^{2}}\left(\frac{I_{\mathrm{Z}}}{\varepsilon}\right), \varepsilon \geq I_{\mathrm{Z}}
$$

where $a_{\mathrm{H}}=6.3 \times 10^{-18} \mathrm{~cm}^{2}$ is the cross-section of the hydrogen photoionization.

The degree of matter $(\mathrm{Fe})$ ionization rises during the expansion and the cloud becomes more transparent. The $\mathrm{K}$-electrons of heavy elements like $\mathrm{Ba}$ still remain connected with nuclei. The optical depth of the cloud due to Ba K-electrons is $\tau=2 h_{\mathrm{Ba}} a_{\mathrm{Ba}} R$. The value $\tau$ $>1$ for quanta with energies $\varepsilon>I_{\mathrm{Ba}}=43 \mathrm{keV}$, when the cloud is transparent for photoabsorption on Fe, if $n_{\mathrm{Ba}} / n_{\mathrm{Fe}} \geq 10^{-3}\left(M_{20} / L_{38}^{2}\right)^{2 / 9}$. Here $M=M_{20} \times 10^{20} \mathrm{~g}$ is the mass of 


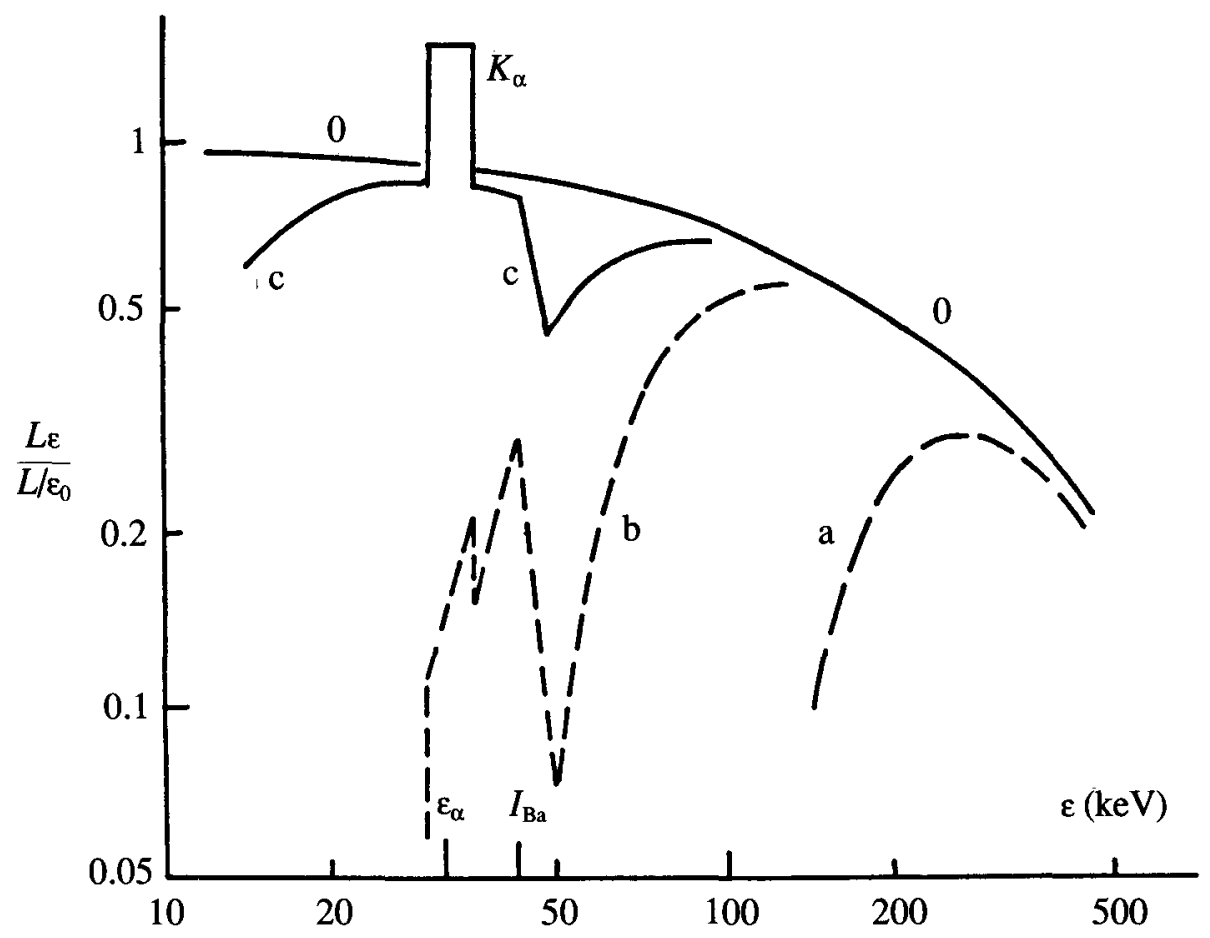

Fig. 3. The time evolution of the absorption line formed in the spectrum of gamma ray burst due to presence of $\mathrm{Ba}$ ions in the Fe plasma of the expanding cloud. The following cloud parameters are chosen: $M_{20}=L_{38}=1, n_{\mathrm{Ba}} / n_{\mathrm{Fe}} \approx 1 / 300$. The time moments are $t_{\mathrm{a}}=t_{1 / 2}$, $t_{\mathrm{b}}=t_{1}=0.45 \mathrm{~s}, t_{\mathrm{c}}=2 t_{1}, 0-$ the initial gamma ray spectrum. At the moment $t_{\mathrm{a}}$ the optical depth to photoabsorption of quanta with energy $\varepsilon=I_{\mathrm{Ba}}=43 \mathrm{keV}$ is equal to $\tau_{01}=60$ on the Fe and $\tau_{1}=9$ on the Ba. At the moment $t_{\mathrm{b}}$ we have $\tau_{01}=1, \tau_{1}=2,3$ and at the moment $t_{\mathrm{c}}$ we have $\tau_{01}=0.015, \tau_{1}=0.6$.

the cloud and $L=L_{38} \times 10^{38} \mathrm{erg} / \mathrm{s}$ is the luminosity. The narrow absorption detail is formed beyond the $\mathrm{K}$-edge of $\mathrm{Ba}$ which is shifted when expansion is included

$$
\varepsilon_{\mathrm{abs}} \approx I_{\mathrm{Ba}}(1+v / c) \approx 50 \mathrm{keV}
$$

A considerable number $(\sim 70 \%)$ of the gamma quanta absorbed by Ba ions are reradiated in the form of the $K_{\alpha}$ line, forming an additional emission feature near $\varepsilon_{\alpha} \approx 30$ (1 $+v / c) \approx 35 \mathrm{keV}$ in the spectrum of gamma ray burst (see Fig. 3). All spectral features gradually disappear during expansion of the cloud. The soft free-free emission of expanding iron plasma is important in the early stages of the burst and may be observed in optics as a short flash with a duration of $\sim 0.5 \mathrm{~s}$ and maximum luminosity $L_{\text {opt }} \approx 5 \times 10^{-4} L_{\gamma}$ (Bisnovatyi-Kogan, Illarionov, 1986). The optical flash must appear in the stage proceeding the formation of spectral features in the gamma band. 


\section{REFERENCES}

Bisnovatyi-Kogan G.S., Chechetkin V.M., 1974, Ap. Sp. Sci., 26, 25.

Bisnovatyi-Kogan G.S., Chechetkin V.M., 1983, Ap. Sp. Sci., 89, 447.

Bisnovatyi-Kogan G.S., Illarionov A.F., 1986, Astron. Zh., 63, 984.

Golenetskii S.V. et al., 1983, preprint F.T.I. N 819.

Mazets E.P. et al., 1980, preprint F.T.I. N 657.

Mazets E.P. et al., 1981, preprint F.T.I. N 719.

Mazets E.P., Golenetskii S.V., 1987, Itogi nauki i techniki, Astronomy, 32, 16.

Radhakrishnan V., 1982, Contemp. Phys. 23, 207. 


\section{DISCUSSION-G. Bisnovatyi-Kogan}

$N$. Itoh: According to your model, $\gamma$-ray bursts are caused by neutron star quakes. Did you investigate the correlation between $\gamma$-ray bursts and pulsar glitches?

G. Bisnovatyi-Kogan: You are right, that pulsar glitches in our model must be correlated with gamma ray bursts. But these bursts must be very faint, because of long distance to the known pulsars. We suppose, that the average distance to the observed gamma ray burst sources is about $100 \mathrm{pc}$. There have been indications of sporadic emission from the Crab pulsar in the region of $30-300 \mathrm{keV}$.

G. Ricker: How would your model account for the two absorption features, one at $\sim 29 \mathrm{keV}$ and the other precisely $2 \times$ higher in energy, recently reported in the same GRB by the Ginga group (Murkami et al 1988)?

G. Bisnovatyi-Kogan: The precision of measurements is not enough for statement that the ratio of energies is precisely two. The observed two lines may be connected with absorption on the two most abundant elements produced in the fission (see fig. 1).

$H$. van der Woerd: From the absorption feature you can make an estimate of the mass of cloud. The cloud has a velocity of $\sim 0.15 \mathrm{C}$, and so you can calculate the energy of the expansion. How does this energy compare to the total energy in the burst?

G. Bisnovatyi-Kogan: In order to overcome the gravitation of the neutron star, the cloud of the mass $\mathrm{M}_{c}$ must obtain kinetic energy of the order of $0.2 \mathrm{M}_{c}{ }^{2}$. If the heat capacity of fission is $\sim 10^{-3} \mathbf{c}^{2}$, then you need $\mathrm{M}_{f}>100 \mathrm{M}_{c}$ to take part in chain reaction. For efficiency of transformation of the thermal energy into kinetic energy, ' $y$ ', the total mass $M_{f}$ $\frac{100 \mathrm{M}_{c}}{y}$ and total energy of explosion $\mathrm{Eb} \sim \frac{0.2 \mathrm{M}_{c} \mathrm{C}^{2}}{\mathrm{y}}$. Almost all this energy, $\mathrm{E}_{b}$ is emitted during gamma-ray burst. For $M=10^{19} \mathrm{~g}, \mathrm{y}=0.3$ we have $\mathrm{E}_{b}=6 \times 10^{39} \mathrm{erg}$. 